\title{
Biotinidase deficiency and infantile epilepsy: Two case reports
}

\author{
*Jithangi Wanigasinghe ${ }^{1}$, N P Perera ${ }^{2}$, N M G L A Gunarathne ${ }^{3}$, E Jasinge ${ }^{4}$
}

Sri Lanka Journal of Child Health, 2015; 44(4): 238-239

(Key words: Biotinidase, epilepsy)

\section{Introduction}

Biotinidase is responsible for the cleavage of biotin from biocytin. Biotin is a cofactor for function of human carboxylases including pyruvate carboxylase, propionyl-coenzyme A (CoA) carboxylase, betamethylcrotonyl-CoA carboxylase, and acetyl-CoA carboxylase. Complete or partial absence of biotinidase is associated with a wide spectrum of clinical manifestations, affecting the neurological, dermatological, immunological and ophthalmological systems $^{1}$. Florid epilepsy is one of the neurological manifestations in the neonatal period or early infancy. The seizures are typically not responsive to conventional anticonvulsant therapies ${ }^{2}$, though rapidly responsive to biotin therapy. We report on two infants with this rare disorder whose epilepsy responded to routine anticonvulsant therapy in spite of early infantile presentation.

\section{Case 1}

A six week-old boy was admitted with multiple daily tonic seizures affecting both upper limbs. Initial electroencephalograms (EEGs) suggested a burst suppression pattern. He was treated with sodium valproate and the seizures responded in a few days. The subsequent EEG showed only intermittent multifocal epileptic discharges in a slow background. Despite seizure control baby had features of encephalopathy and marked developmental delay.

${ }^{1}$ Consultant Paediatric Neurologist and Senior Lecturer in Paediatrics, Faculty of Medicine, University of Colombo, ${ }^{2}$ Senior Registrar in Neurology, ${ }^{3}$ Registrar in Paediatrics, ${ }^{4}$ Chemical Pathologist, Lady Ridgeway Hospital for Children, Colombo

*Correspondence: jithangi@gmail.com

(Received on $1^{\text {st }}$ August 2014: Accepted after revision on 19 September 2014)

The authors declare that there are no conflicts of interest

Open Access Article published under the Creative

Commons CC-BY-NC-ND Licence.

Examination revealed severe seborrheic dermatitis on his scalp (Figure 1) and perineal region.

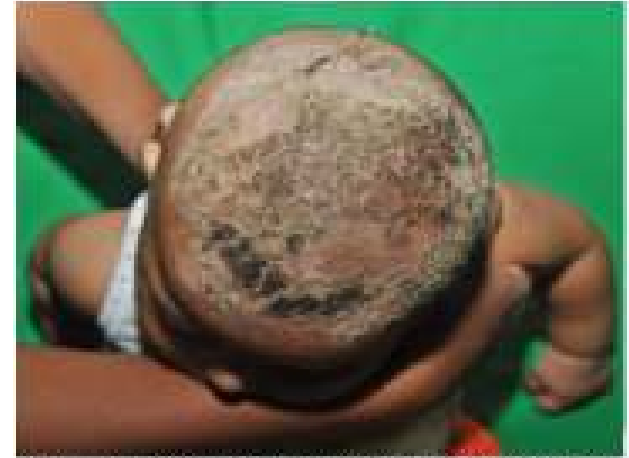

Figure 1: Seborriteic dermatinis before treatmeat

*Permission given by parents to publish photograph

He was a product of consanguinity with death of a sibling in infancy due to unexplained, rapidly progressive septic illness. During a febrile illness he was detected to have a metabolic acidosis with normal anion gap and normal ammonia hinting of multiple carboxylase deficiency. The Guthrie card screening showed low biotin levels. Since biotinidase enzyme level assay was not affordable to the parents, he was commenced on therapeutic trial of biotin. The response to therapy was immediate with disappearance of the encephalopathic behaviour, improvement of the seborrheic dermatitis (Figure 2), and improved visual responsiveness.

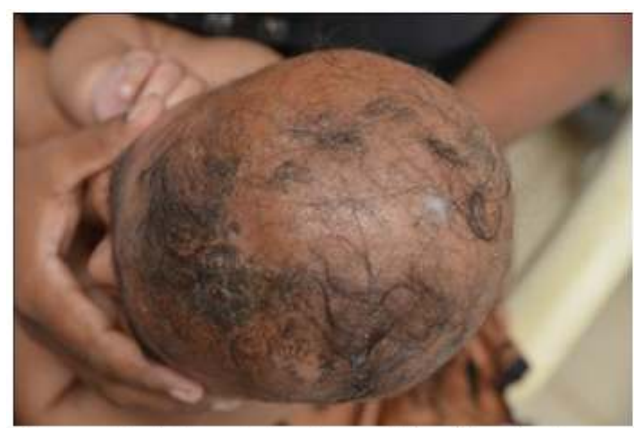

Figure 2: Seborrheic dermatitis after treatment

*Permission given by parents to publish photograph

In order to confirm biotinidase deficiency, the biotin was taken off after 2 weeks of therapy. This resulted 
in reappearance of the encephalopathic behaviour and the dermatitis but no seizures. Recommencement of biotin resulted in reversal of all the above clinical features. When reviewed at four months of age, he continued to be seizure free, have normal visual responsiveness, early vocalization, and social smile and attempts to control the head.

\section{Case 2}

An 8 week old baby boy presented with focal seizures manifesting as right arm extension followed by bilateral convulsive movements occurring as small clusters every 1-2 weeks. Initial EEG was normal. Seizures became infrequent on a combination of carbamazepine, topiramate and leveteracetam. However, he continued to have unusual persistent drowsiness. Examination revealed only microcephaly but no skin rashes. Development was static with head lag and no social smile or vocalisation at 3 months of age. Two severe respiratory tract infections occurred during the next two months.

At four months, he developed epileptic spasms without clustering. The EEG showed a hypsarrhythmia. These single spasms dramatically responded to oral prednisolone. However, he continued to be in an encephalopathic state. Investigations revealed normal blood gas and ammonia levels. Computed tomography of brain showed increased extra axial space with patchy hypodense areas in deep white matter. Metabolic screening on dried spot blood indicated biotinidase deficiency, confirmed by biotinidase enzyme level of $1.42 / \mathrm{min} / \mathrm{ml}$ (normal $>5 / \mathrm{min} / \mathrm{ml}$ ) indicating severe enzyme deficiency. Immediate improvement of drowsiness, development of social smile and early vocalization was noted within two weeks of treatment with biotin $10 \mathrm{mg}$ daily. Review over next two months confirmed seizure freedom, absence of any further respiratory infections, and further improvement in development with achievement of head control at 6 months of age.

\section{Discussion}

Absence or deficiency of biotinidase impairs the cleavage of biotin from biocytin causing a deficiency of free biotin, slowing the functioning of biotindependent carboxylases. This impairment causes abnormalities in fatty acid synthesis, amino acid catabolism, and gluconeogenesis which may manifest as various clinical findings such as vomiting, lethargy and hypotonia, skin rash, conjunctivitis, alopecia, ataxia and increased susceptibility to infections. Laboratory findings include a ketolactic acidosis and organic aciduria. Dried blood spot test for biotinidase levels can be used as a screening test; however confirmatory test is based on biotinidase activity assessment in serum. Profound biotinidase deficiency $(<10 \%$ of normal serum enzyme activity) as in our second infant typically presents in the first 6 months of life. Although we had no enzyme assay in the other patient, the diagnosis was established based on positive screening test and dramatic reappearance of clinical features that responded to therapy on withdrawal.

The seizures in biotinidase deficiency are considered to be unresponsive to conventional antiepileptic drugs $^{3}$. However, our two patients showed a good response. They did not experience florid, refractory epilepsy. Their diagnosis was prompted by the early onset and persistent encephalopathy, clinical history, seborrheic dermatitis and supportive biochemistry.

This case history highlights the importance of vigilance on possible treatable epileptic encephalopathies in infantile epilepsies. Treatment is with oral biotin at a dose of $10 \mathrm{mg}$ /day which is adequate to treat even profound deficiency, thus biotinidase deficiency is included in the universal newborn screening programmes. The prognosis is good if treatment is initiated early and continued lifelong ${ }^{4}$.

\section{References}

1. Wolf B. Clinical issues and frequent questions about biotinidase deficiency. Molecular Genetics and Metabolism 2010; 100(1):6-13. http://dx.doi.org/10.1016/j.ymgme.2010.01. 003

PMid: 20129807

2. Karimzadeh P, Ahmadabadi F, Jafari N, Jabbehdari S, Alaee MR, Ghofrani M, et al. Biotinidase deficiency: a reversible neurometabolic disorder (an Iranian pediatric case series). Iranian Journal of Child Neurology 2013; 7(4):47-52. PMid: 24665318 PMCid: PMC3943044

3. Wolf B. The neurology of biotinidase deficiency. Molecular Genetics and Metabolism 2011; 104(1-2):27-34. http://dx.doi.org/10.1016/j.ymgme.2011.06. 001

PMid: 21696988 
4. Bousounis DP, Camfield PR, Wolf B. Reversal of brain atrophy with biotin treatment in biotinidase deficiency. Neuropediatrics 1993; 24(4):214-7.

http://dx.doi.org/10.1055/s-2008-1071543

PMid: 8232780 